\title{
Switching of Geometric Phase in Degenerate Systems
}

\author{
A. Yahalom ${ }^{a, b}$ and R. Englman ${ }^{b, c}$ \\ ${ }^{a}$ Faculty of Engineering, \\ Tel-Aviv University, Tel-Aviv 69978, Israel \\ ${ }^{b}$ Research Institute, \\ College of Judea and Samaria, Ariel 44284, Israel \\ ${ }^{c}$ Department of Physics an Applied Mathematics, \\ Soreq NRC,Yavne 81810, Israel \\ e-mail: asya@post.tau.ac.il, englman@vms.huji.ac.il
}

November 21, 2018

\begin{abstract}
The geometric and open path phases of a four-state system subject to time varying cyclic potentials are computed from the Schrödinger equation. Fast oscillations are found in the non-adiabatic case. For parameter values such that the system possesses degenerate levels, the geometric phase becomes anomalous, undergoing a sign switch. A physical system to which the results apply is a molecular dimer with two interacting electrons. Additionally, the sudden switching of the geometric phase promises to be an efficient control in two-qubit quantum computing.
\end{abstract}

PACS: 03.65.Ge; 03.67.Lx; 76.60.-k

keywords: Berry's phase; Non-adiabaticity; Quantum computation

\section{Introduction}

Following the discovery of the topological (or geometric) phase acquired by a system that evolves adiabatically around a closed path in a parameter space [1]-[3], many instances of the phenomenon have been found either theoretically or in experiments [⿶]-[7]. A widespread quantum mechanical 
application of the theory is when an electron in a molecule slowly evolves subject to a field due to (say) the host ions, such that the field varies periodically in time. In typical cases the electronic state changes sign after a full period [1]. It is entirely obvious that for a pair of electrons, which are independent except that both are subject to fields with the same period (though the fields may have otherwise different characters) the total electronic wave function (being a product of two individual wave functions) will return to its original value after a period. Similarly, when the field on the second electron is a constant or has a period which is $\frac{1}{2}$ or $\frac{1}{4}$ (and so on) times the period for the first field then, after one complete revolution, the total wave function will again acquire a change of sign. It is of interest to consider next what happens to the geometrical phase if one introduces an interaction between the two electrons. One would expect that the geometrical phase would not be altered by the interaction. This is indeed the case for the model studied in this work, with one notable exception: When the two-electron states become degenerate by some coincidence of the parameters in the Hamiltonian, then under suitable conditions the expected sign change becomes reversed. This phenomenon is studied in this work. Other aspects of states that have degeneracies on or off the trajectories have been noted earlier in [8, 9]. The topological phase change after a full revolution has been interpreted in several previous publication as a surface integral in the parameter

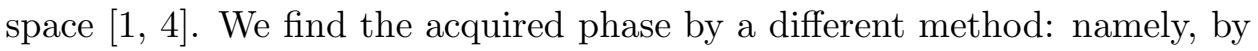
numerical integration of the time dependent Schrödinger equation, which is the appropriate method for circumstances that the surface integral is not readily available. Moreover, this method, which was previously used for a single electron 110, 11, 12], gives the entire open path phase, rather then the geometrical phase alone. Theoretical expressions and interpretations for the open path phase were given in [13, 14]. The last section contains algebraic and numerically less convenient calculations.

\section{The physical model}

Brief descriptions of the model have been given previously [15, 16]. In essence, we consider a dimer consisting of two vibrating, planar and possibly dissimilar molecules lying upon each other. A simple instance is two triatomic molecules $A_{3}-B_{3}$. In addition to several tightly bound electrons, each molecule contains one electron that is in an orbital doublet state, degenerate in the symmetric (equilateral triangle) configuration of its host. 
The molecules are sufficiently remote for the orbital doublets to be localized on the host molecule and also for overlap (and exchange) effects to be negligible. There remain vibronic interactions between the electronic and the in-plane vibrational motions, as well as an inter-electronic coupling. The former (Jahn-Teller) interaction [17] is conveniently treated by the formalism of Longuet-Higgins et al [18], which represents the two electronic states by $e^{ \pm i \theta_{m}}$, where $\theta_{m}$ an angular variable and $m=1,2$ for the two molecules. In this work we shall modify this formalism by using as the basis the direct product of two real representations, namely $\cos \theta_{1} \cos \theta_{2}$, etc. The non-totally symmetric vibrational mode coordinates of the molecules are denoted by $q_{m} \cos \phi_{m}$ and $q_{m} \sin \phi_{m}$ [17]. In terms of the electronic and vibrational coordinates, as given above, and the vibronic coupling strengths $G_{m}$, the interaction may be simply expressed as

$$
H_{m}=G_{m} \cos \left(2 \theta_{m}-\phi_{m}\right) \quad m=1,2
$$

¿From elementary physical considerations, the vibronic constants $G_{m}$ are (at least approximately) proportional to the mode amplitudes $q_{m}$. The interaction between electrons on the two molecular entities is familiar from energy transfer and other subjects, and is expressed by terms that contain jointly excitation and de-excitation operators on two molecules [19]. For simplicity we assume for the interaction term the large $U(t / U \rightarrow 0)$," antiferromagnetic Heisenberg" limit of the well-known Hubbard Hamiltonian [20], having the product form

$$
H_{12}=2 G \cos \left(2 \theta_{1}-\phi_{1}\right) \cos \left(2 \theta_{2}-\phi_{2}\right)
$$

This is symmetric and even in the relative angular variables and is invariant under a full rotation of either mode angular variable $\phi_{m}$. Analogously to Hubbard's $U$ which gives the exchange splitting between like and unlike spins on the same site, $G$ represents the electrostatic splitting between like and unlike vibronic states on the two molecules. $G$ depends foremost on the distance between the molecules and only weakly on the molecular displacement coordinates. In previous works [15, 16] the cases $G_{m}$ larger and smaller than $G$ were studied separately.(To exhibit formally the weakness of overlap effects, one would introduce, both in the wave functions and in the couplings, two more factors that depend on two further coordinates, (say) $r_{1}$ and $r_{2}$, such that the overlaps between the $r_{1}$ and $r_{2}$ factors are negligible.)

The total Hamiltonian $H$ is the sum of equations(11) and (2).

$$
H=H_{1}+H_{2}+H_{12}
$$


We next represent the vibrations of the molecules as rotational motions in the $(q, \phi)$-planes. (The potential surfaces in Figure 3.3 of [17 admit of such motion.) Classically, this is equivalent to taking $q_{1}=$ constant, $q_{2}=$ constant, $\phi_{1}=\omega_{1} t, \phi_{2}=\omega_{2} t$, where $t$ is time and $\omega_{1}$ and $\omega_{2}$ are the angular frequencies of the (small) molecular displacements. The equation that forms the basis of this work is the time dependent Schrödinger equation, with $\hbar=1$,

$$
i \frac{\partial \Psi}{\partial t}=H \Psi
$$

The two-electronic product states are labeled as follows:

$$
\begin{aligned}
& \psi_{1}=N \cos \theta_{1} \cos \theta_{2} \\
& \psi_{2}=N \sin \theta_{1} \cos \theta_{2} \\
& \psi_{3}=N \cos \theta_{1} \sin \theta_{2} \\
& \psi_{4}=N \sin \theta_{1} \sin \theta_{2}
\end{aligned}
$$

where the normalization constant, $N=\frac{1}{\pi}$. In this basis the Hamiltonian finds the following representation:

$$
\begin{aligned}
& H=\frac{1}{2} \times \\
& \left(\begin{array}{cccc}
G_{1} \cos \phi_{1}+G_{2} \cos \phi_{2} & G_{1} \sin \phi_{1}+G \sin \phi_{1} \cos \phi_{2} & G_{2} \sin \phi_{2}+G \cos \phi_{1} \sin \phi_{2} & G \sin \phi_{1} \sin \phi_{2} \\
+G \cos \phi_{1} \cos \phi_{2} & -G_{1} \cos \phi_{1}+G_{2} \cos \phi_{2} & G \sin \phi_{1} \sin \phi_{2} & G_{2} \sin \phi_{2}-G \cos \phi_{1} \sin \phi_{2} \\
G_{1} \sin \phi_{1}+G \sin \phi_{1} \cos \phi_{2} & -G \cos \phi_{1} \cos \phi_{2} & G_{1} \cos \phi_{1}-G_{2} \cos \phi_{2} & G_{1} \sin \phi_{1}-G \sin \phi_{1} \cos \phi_{2} \\
G_{2} \sin \phi_{2}+G \cos \phi_{1} \sin \phi_{2} & G \sin \phi_{1} \sin \phi_{2} & -G \cos \phi_{1} \cos \phi_{2} & \\
G \sin \phi_{1} \sin \phi_{2} & G_{2} \sin \phi_{2}-G \cos \phi_{1} \sin \phi_{2} & G_{1} \sin \phi_{1}-G \sin \phi_{1} \cos \phi_{2} & -G_{1} \cos \phi_{1}-G_{2} \cos \phi_{2}
\end{array}\right)
\end{aligned}
$$

The eigen energies of this matrix, denoted by $\kappa_{r}^{A D}(r=1, . ., 4)$ and the corresponding eigen functions (the so-called adiabatic eigen states) are:

$$
\begin{array}{lll}
\kappa_{1}^{A D}=\frac{1}{2}\left(G_{1}+G_{2}+G\right) & \rightarrow \psi_{1}^{A D}=\frac{1}{\pi} \cos \left(\theta_{1}-\frac{\phi_{1}}{2}\right) \cos \left(\theta_{2}-\frac{\phi_{2}}{2}\right) \\
\kappa_{2}^{A D}=\frac{1}{2}\left(-G_{1}+G_{2}-G\right) & \rightarrow \psi_{2}^{A D}=\frac{1}{\pi} \sin \left(\theta_{1}-\frac{\phi_{1}}{2}\right) \cos \left(\theta_{2}-\frac{\phi_{2}}{2}\right) \\
\kappa_{3}^{A D}=\frac{1}{2}\left(G_{1}-G_{2}-G\right) & \rightarrow \psi_{3}^{A D}=\frac{1}{\pi} \cos \left(\theta_{1}-\frac{\phi_{1}}{2}\right) \sin \left(\theta_{2}-\frac{\phi_{2}}{2}\right) \\
\kappa_{4}^{A D}=\frac{1}{2}\left(-G_{1}-G_{2}+G\right) & \rightarrow \psi_{4}^{A D}=\frac{1}{\pi} \sin \left(\theta_{1}-\frac{\phi_{1}}{2}\right) \sin \left(\theta_{2}-\frac{\phi_{2}}{2}\right)
\end{array}
$$




\section{Time evolution}

Recalling that $\phi_{1}=\omega_{1} t$ and $\phi_{2}=\omega_{2} t$, we substitute the time dependent Hamiltonian matrix, equation (6), into equation (4) to obtain the solution $\Psi$ (which is now a four column-vector) by forward integration, using a "Mathematica" numerical algorithm. With the choice of $\omega_{1}=1$ and $\omega_{2}$ a simple multiple of $\omega_{1}$, the periodicity is $2 \pi$ and the solutions exhibited in the following figures stretch over a single period of the Hamiltonian, $t=0-2 \pi$. Beyond this, the solutions can be obtained by symmetry. The initial value of $\Psi$ at $t=0$ was taken as $(1,0,0,0)$. This choice implies no loss of generality, since any other solution can be derived from this through a change of the parameters, shift of the time scale and superposition of solutions. The analytic treatment of the Schrödinger equation including the interaction, given in the last section, involves the solution of a quartic equation which is not decomposable into two quadratic equations. This stands in contrast to the one-electron two- state problem, whose Schrödinger equation involves the solution of a quadratic [21, 22, 12]. Thus the interaction introduces in the problem a genuinely new element.

(A) Non-adiabatic effects.

We first investigate the approach to the adiabatic limit, which is the condition under which the results of [1] were derived. To achieve this limit, at least one of the coupling coefficients $G_{1}, G_{2}$ and $G$ has to be large. (Basically, their ratio to omega serves as the measure of adiabaticity [11]. We define it by $\left.A=\left|\frac{G_{1}}{\omega_{1}}\right|\right)$ The approach to adiabaticity is exemplified in Figure 1, whose two curves have identical parameters except that all coupling strengths are reduced by a factor 10 in the thinly drawn curve, thus not yet reaching the adiabatic limit.

In either curve there is a sign change in the wave function at full revolution, but the lack of adiabaticity shows up in two ways: First, in the state amplitudes it induces oscillations, whose periods depend on the coupling strength. These oscillations are the ionic-field analogues of the Rabi oscillations (that are due to excitations by an electro-magnetic field). The former have been analyzed in detail in an earlier paper on a two-state model [12]. Secondly, the state amplitude does not reach -1 , but only approximately -.75. This reduction has already been quantitatively explained for the two state model in [10] and will not be further discussed here. In the fully adiabatic limit, the solution can be read off from the analytic expressions given in equation (7) . Having started (by virtue of the initial conditions imposed) with $\psi_{1}^{A D}$, we see that the thick curve shown in Fig. 1 indeed approximates 


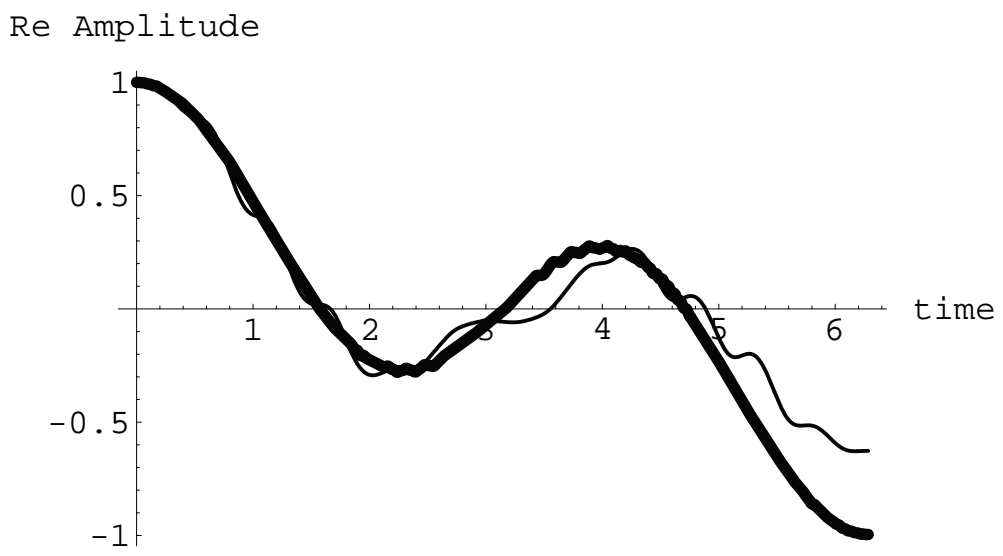

Figure 1: Non-adiabaticity effects in the real part of the initially excited component, as a function of time. The frequencies on the two dimers are $\omega_{1}=1$ and $\omega_{2}=2$. The values of the coupling parameters are as follows. Thick line: $G_{1}=-80, G_{2}=-160, G=40$ (near adiabatic limit). Thin line: $G_{1}=-8, G_{2}=-16, G=4$ (non-adiabatic case)

. The dynamic phase has been subtracted in this and in the following figures.

well to the expression $\cos (t / 2) \cos (t)$, which is the amplitude of the first component in $\psi_{1}^{A D}$, according to equation (5) and equation (7) . Further solutions for the near-adiabatic limit are shown graphically in Figures 2 and 3.

(B) Enters degeneracy.

Further computations in the adiabatic limit (large $G$ 's) with other parameters likewise confirm the expressions in equation (7), as predicted by the adiabatic theorem. As is well known, this theorem is based on the noncrossing of energy levels [23]. In the absence of inter-electronic coupling, the two electrons move independently and, therefore, even if there was a degeneracy in the system through the combination of the levels, no effect would be felt. For a coupled two-electron system, the degeneracy is expected to produce new effects.

This was indeed found. Thus, when in equation (2) the inter-electron coupling was so adjusted that

$$
G=-G_{1}
$$

then the first two levels in equation (7) become degenerate. The accompanying curves (a)-(c) in Figure 4 show the way the amplitude (of the first component) changes as this degeneracy is traversed through subsequent sequence of changes in $G$. The results are similar when the changes are made 


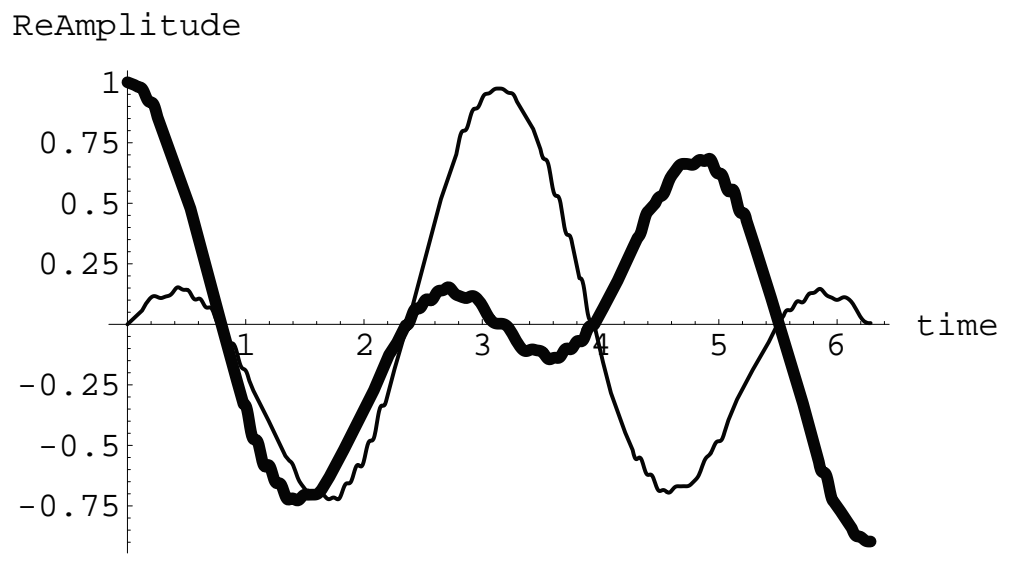

Figure 2: Two-electron state amplitudes in a dimer. The thick line shows the time dependent amplitude of the first (initially excited component), the thin line that of the second component in Equation (5). Frequencies: $\omega_{1}=1$, $\omega_{2}=4, G_{1}=-40, G_{2}=-80, G=16$ (near adiabatic limit)

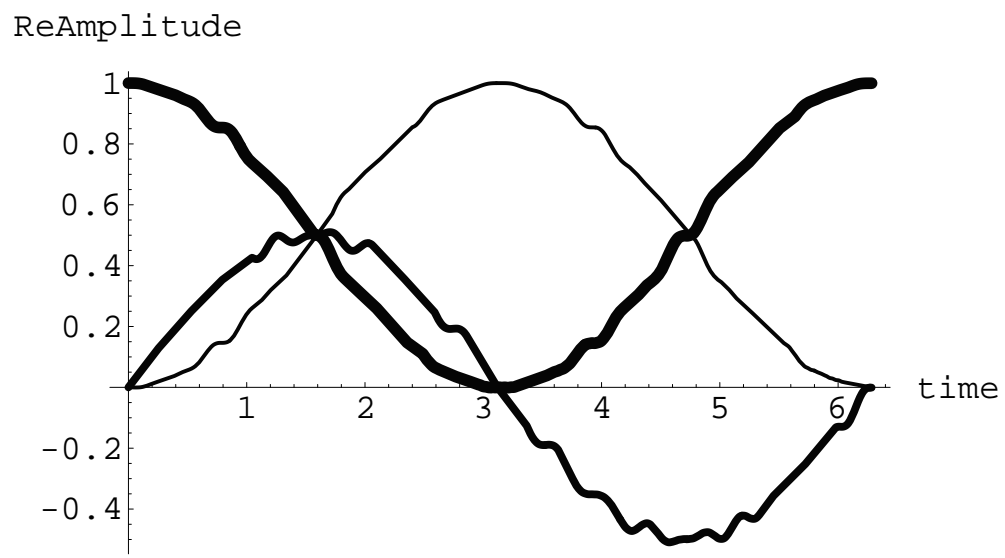

Figure 3: Two-electron state amplitude in a dimer, with both molecules subject to a periodic force. After a full revolution the two electronic states each change their sign, leaving the total state invariant. Frequencies: $\omega_{1}=1$, $\omega_{2}=1, G_{1}=-100, G_{2}=-200, G=40$ (near adiabatic limit). Thick line: First, initially excited component. Medium thick line: Second and third components. Thin line: Fourth component. 


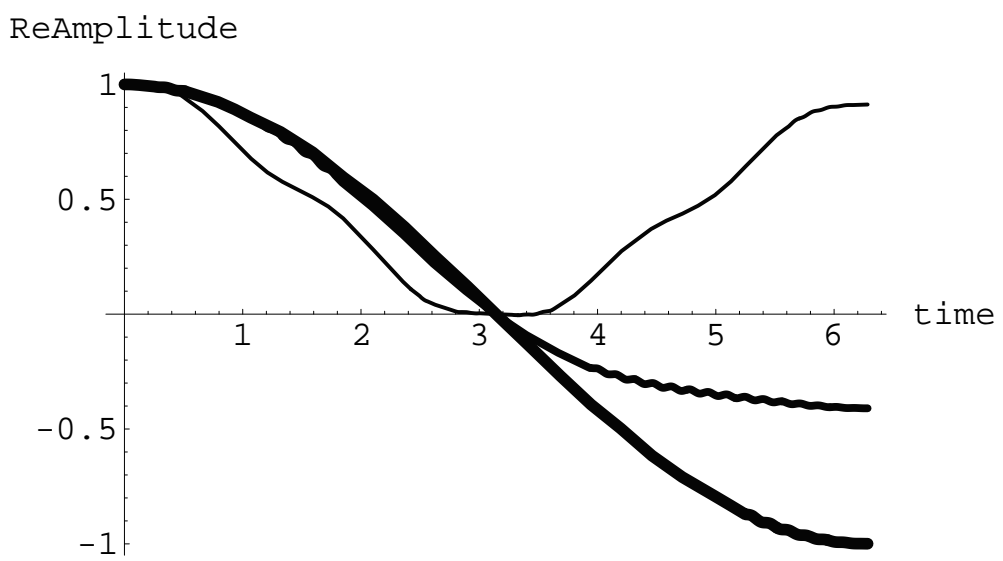

Figure 4: Degeneracy and near-degeneracy effects in the first, initially excited component.The following values are common to the three curves: $\omega_{1}=1, \omega_{2}=1, G_{1}=-40, G_{2}=-80$. The inter-electronic coupling $G$ is varied as follows: Thin-line: $G=36$ and 44 (non-degenerate). Medium thick line: $G=39.6$ and 40.4 (near degenerate case). Thick line: $G=40$ (exact degeneracy).

in an electron-vibration coupling strength $G_{1}$. The curves are computed in the near-adiabatic limit.

One observes that with degenerate parameters, the amplitude keeps its sign at a half period and achieves at full revolution a switched sign with respect to that in the non-degenerate situation. The switch occurs abruptly as the coupling strength(s) is (are) varied. We now turn to a discussion of this switch, regarding its dependence on adiabaticity and its stability, an application and the algebraic treatment of the results.

\section{Discussion}

The preceding figure (fig. 4) was plotted in the near adiabatic limit when $A=\left|\frac{G_{1}}{\omega_{1}}\right|$ is large $(=40)$.In the extreme adiabatic limit when $A=\infty$ only the symmetric ( thin ) line (ending at +1 ) and the antisymmetric (thick) line (ending at -1 ) are possible, the latter occurring at exact degeneracy and the former for any values of the parameters that do not cause exact degeneracy. When the adiabaticity parameter $A$ is finite, though large, for large deviation from degeneracy the computed curve approaches the symmetric curve and for small values of the deviation, the antisymmetric curve. It is possible to describe this effect quantitatively, by finding a relation between the adiabaticity $A$ and that value of the deviation from precise degeneracy 
at which the real part of the amplitude at full revolution (at time $=2 \pi$ ) is zero (i.e., just half way between the values far from and exactly at degeneracy). Attaching to this value of the deviation from degeneracy the subscript 0 , we find from our computation for the present model

$$
\left|\left(G+G_{1}\right)_{0}\right|=0.54 \frac{\left|G_{1}\right|}{A}
$$

(We recall that at $G+G_{1}=0$ one has a strict degeneracy.) For a numerical illustration of this result, when the adiabaticity $A=40$ (this being the absolute value of the ratio of the coupling strength $G_{1}$ to the angular frequency of motion $\omega_{1}$, as e.g. when $G_{1}=-40, \omega_{1}=1$ ), then a deviation from degeneracy less than 0.54 will cause in the phase a switch whose magnitude is at least $\frac{\pi}{2}$.In fig. 4, the thin line is for a deviation 4 that is larger than 0.54 and the phase switch is close to zero, the medium thick line is for a deviation of .4 , which is below the 0.54 limit and the phase switch is larger than $\frac{\pi}{2}$. Though equation (9) is derived from numerical fitting (of over one and a half orders of magnitude in $A$ and to an average accuracy of about five percent) for a specific model, we expect that a qualitatively similar relation would hold in other cases where the degeneracy causes a switch in the phase. A deviation from (or removal of) the degeneracy can also achieved by adding off-diagonal terms $\delta H_{12}=\delta H_{21}$ in the $(1,2)$ and $(2,1)$ positions to he Hamiltonian matrix of equation(6). When these perturbation terms are constants their effect on the switching is given very closely by equation (9) , when the deviation on the left hand side stands for $\delta H_{12}$, or the added matrix element.This represents a test for the stability of the switch against a small deviation from the special form of the interaction assumed in this work. (It would be tempting to combine the two findings into a single formula, in which the left hand side of equation (9) is simply the splitting of the two near degenerate levels, given by

$$
\text { splitting }=\left(\left(G+G_{1}\right)_{0}^{2}+4\left(\delta H_{12}\right)^{2}\right)^{\frac{1}{2}}
$$

but our numerical results indicate that the above formula holds without the factor 4.)

A further stability test was also made: When a constant was added to each of the terms in top-right to bottom-left diagonal, the computed curves were not changed in a perceptible way, even when the constant reached .2 of the (root mean square) value of the term. 
A vibrating dimer, carrying one electron on either molecule, was used as a model system to introduce the formalism. We now turn to an alternative application.

The four state formalism in this paper can be naturally interpreted as a two-qubit system. "Qubits" are two-state building-blocks for quantum computing. (An introductory account is found in [24].) The two-qubit situation is the minimal one to describe logical operations between control and target [25, 26], and it has also been shown to be sufficient for that purpose [27]. For an identification of logical operations in a two-qubit scheme, one can refer to 28]. The correspondence between the time dependent description (or the Schrödinger equation (4), on which the present work is based) and quantum computing has been developed in [29]. One of the several possible implementation is the use of trapped ions in doublet quantum states [30]. as a control-no gate. This may be done as follows. We have seen that as the first and second components in equation (]$\left.^{7}\right)$ are brought to degeneracy through the choice $G \rightarrow-G_{1}$, then (upon full revolution) there is a sign-switch in the first component in equation (7) . Likewise, in the second (co-degenerate) component. There is, however, no switch in the third or in the fourth components (which are energetically distinct). Suitable combinations (e.g., sum and difference) of the first two components can thus be used for representation of "Yes" and "No" operations, with the choice of the coupling parameters acting as control. One important result of this paper is the abrupt switching of the geometric phase through a slight change in the coupling strength, as shown in Figure 4, and this can be conveniently used for manipulation on the target, or of the outcome, by external control. The abruptness of the switching is of some additional interest, in that the change in the total phase of the target, upon a slight change of the parameter, is more due to the geometric phase than to the change in the dynamic phase. This means that the dynamic phase need not necessarily be subtracted from the total phase. Abrupt switching of the Pancharatnam phase for two polarized light beams was described and observed earlier [31].

Further extension of our results might involve consideration of several (more than two) qubit states. This may be applicable to quantum computation, in which an important issue is how to achieve reduction of decoherence of phases by random causes. It has thus been proposed that a possible way is the use of superposition states composed of several qubits [32, 24]. 


\section{$5 \quad$ Algebraic treatment}

The solutions of the Schrödinger equation (四) and equation (6), obtained in the text numerically, can be exhibited through a sequence of(three) matrix multiplications operating on a column (four) - vector $\vec{\chi}(t)$ which contains the initial conditions. The vector is written in the representation of equation (6) as

$$
\vec{\chi}(t)=\left(\begin{array}{c}
e^{-i \kappa_{1} t} \chi_{1}(0) \\
e^{-i \kappa_{2} t} \chi_{2}(0) \\
e^{-i \kappa_{3} t} \chi_{3}(0) \\
e^{-i \kappa_{4} t} \chi_{4}(0)
\end{array}\right)
$$

and contains initial values needed to yield the initial values of the solution and the eigen frequencies $\kappa_{r}$ (of the Rabi-type) of a four matrix. In the extreme adiabatic limit and excluding degeneracy. the eigen frequencies can be given explicitly and are identical with the eigenvalues $\kappa_{r}^{A D}$ shown in equation (7) . Non-adiabatic corrections to the (non-degenerate) eigenvalues are of the order of $\left(\frac{\omega}{G}\right)^{2}$. The full solution is

$$
\Psi=\mathcal{M F} \mathcal{A} \vec{\chi}(t)
$$

The matrix $M$ generates a complex representation when applied to the real basis shown in equation (5). Its form is

$$
\mathcal{M}=\frac{1}{2}\left(\begin{array}{cccc}
1 & i & i & -1 \\
1 & -i & i & 1 \\
1 & i & -i & 1 \\
1 & -i & -i & -1
\end{array}\right)
$$

The next matrix $\mathcal{F}$ is diagonal and contains time exponents with the coefficient $\frac{1}{2}$, which is the source of the sign change upon a full revolution.

$$
\mathcal{F}=\left(\begin{array}{cccc}
e^{-\frac{i}{2}\left(\omega_{1}+\omega_{2}\right) t} & 0 & 0 & 0 \\
0 & e^{\frac{i}{2}\left(\omega_{1}-\omega_{2}\right) t} & 0 & 0 \\
0 & 0 & e^{\frac{i}{2}\left(-\omega_{1}+\omega_{2}\right) t} & 0 \\
0 & 0 & 0 & e^{\frac{i}{2}\left(\omega_{1}+\omega_{2}\right) t}
\end{array}\right)
$$

Lastly, $\mathcal{A}$ diagonalizes the following matrix with the eigenvalues $\kappa_{r}(r=$ $1, . .4)$ appearing above. 


$$
H^{s t}=\frac{1}{2}\left(\begin{array}{cccc}
-\omega_{1}-\omega_{2} & G_{1} & G_{2} & G \\
G_{1} & \omega_{1}-\omega_{2} & G & G_{2} \\
G_{2} & G & -\omega_{1}+\omega_{2} & G_{1} \\
G & G_{2} & G_{1} & \omega_{1}+\omega_{2}
\end{array}\right)
$$

To prove equation equation (12) substitute into the Schrödinger equation (4) and transpose the time derivative of the factor $\mathcal{F}$ in equation (14) from the left to the right hand side. In the adiabatic limit, when the frequencies are vanishingly small compared to the coupling strengths, $\mathcal{A}$ simplifies to

$$
\mathcal{A}=\frac{1}{2}\left(\begin{array}{cccc}
1 & 1 & 1 & 1 \\
1 & -1 & 1 & -1 \\
1 & 1 & -1 & -1 \\
1 & -1 & -1 & 1
\end{array}\right)
$$

When some of the adiabatic eigenvalues approach degeneracy, e.g.,

$$
\kappa_{2}^{A D} \rightarrow \kappa_{1}^{A D}
$$

the situation changes dramatically. The corresponding non-adiabatic eigenvalues differ from the adiabatic ones by a term that is linear in a frequency. Specifically, to a first order in the $\omega$ 's,

$$
\kappa_{s}-\kappa_{s}^{A D}= \pm \frac{1}{2} \omega_{1} \quad(s=1,2)
$$

This half-frequency value in the $\kappa$-exponents shown in equation (11) adds on a further factor of -1 upon a full revolution, thus accounting for the sign switch found in the degenerate situation. Also (as the numerical solutions show), two components in the four-vector become vanishingly small throughout the time-range. With the initial conditions chosen for our solution, the vanishing components are the second and the fourth. If we remove the corresponding rows and columns from the Hamiltonian shown in equation (6) , the resulting two-by-two matrix becomes under the degeneracy condition $G_{1}=-G$,

$$
\begin{aligned}
H & =\frac{1}{2}\left(\begin{array}{cc}
\left(G_{2}+G \cos \phi_{1}\right) \cos \phi_{2} & \left(G_{2}+G \cos \phi_{1}\right) \sin \phi_{2} \\
\left(G_{2}+G \cos \phi_{1}\right) \sin \phi_{2} & -\left(G_{2}+G \cos \phi_{1}\right) \cos \phi_{2}
\end{array}\right) \\
& -\frac{1}{2} G \cos \phi_{1} \quad \text { (a scalar) }
\end{aligned}
$$


Since $\left(G_{2}+G \cos \phi_{1}\right)$ is a common factor for all elements in the matrix, it does not enter the component amplitudes and these contain only the angle $\phi_{2}$. This represents a second algebraic justification for the phase switch upon degeneracy.

The relation in equation (8) represents a three dimensional surface of degeneracy in the four dimensional displacement-manifold [15, 16]. Actually, the surface is (as expected) only two dimensional, for the following reason: The degeneracy involves states 1 and 2. An off-diagonal coupling between these states would lift the degeneracy, even if this coupling would be so weak as not to affect the dynamic behavior. By our ignoring this coupling, we effectively put it equal to zero, which represents a further relation between some of the coordinates, say, between $q_{1}$ and $q_{2}$.In summary, we have investigated both the geometric phase (i.e., at full revolution) and the interim open path phases for two interacting electrons, each in a doublet state, when they move in cyclically varying potentials. With the choice of a product inter electron potential, the eigen energies are constants along the motion. The dynamic phase (being the time integral of an eigen energy) can thus be conveniently subtracted off from the total phase. With more general interactions, one would need a more complicated procedure for subtraction. We have identified non-adiabatic effects (namely, high frequency oscillations and the incomplete return to the initial state after a full revolution). We then elucidated a switch of the sign of the wave-function as the system develops a degeneracy in the two-electron energy levels. The switch

was shown to be quite sudden as the degeneracy situation was approached through varying the parameters in the Hamiltonian.

\section{Acknowledgment}

The authors thank Professor Alden Mead for critical readings of the manuscript and Dr. Tal Mor for discussing some computing aspects.

\section{References}

[1] M.V. Berry, Proc. Roy. Soc. (London)A 39245 (1984)

[2] B. Simon, Phys. Rev. Letters 512167 (1983)

[3] Y. Aharonov and J. Anandan, Phys. Rev. Letters 581593 (1987) 
[4] A. Shapere and F. Wilczek (editors), Geometric Phases in Physics (World Scientific, Singapore, 1989)

[5] J. Zak, Phys. Rev. Letters 522747 (1989)

[6] H. von Busch, Vas Dev, H. - A. Eckel, S. Kasahara, J. Wang, W. Demtroeder, P. Sebald and W. Meyer, Phys. Rev. Letters 814584 (1998)

[7] J.W. Zwanziger, S.P. Rucker and G.C. Chingas, Phys. Rev. A 433232 (1991)

[8] F. Wilczek and A. Zee, Phys. Rev. Letters 522111 (1984)

[9] W.D. Heiss, Eur. Phys. J. D 71 (1999)

[10] R. Englman, A. Yahalom and M. Baer, Phys. Letters A 251223 (1999)

[11] R. Englman and A. Yahalom, Phys. Rev. A 601890 (1999)

[12] R. Englman, A.Yahalom and M. Baer, Eur. Phys. J. D 81 (2000)

[13] S. R. Jain and A.K. Pati, Phys. Rev. Letters 80650 (1998)

[14] A.K. Pati, Phys. Rev. A 522576 (1995); Phys. Rev. A 60 121(1999)

[15] M. Baer, A.J.C. Varandas and R. Englman, J. Chem. Phys. 1119493 (1999)

[16] M. Baer, R. Englman and A.J.C. Varandas, Mol. Phys. 971185 (2000)

[17] R. Englman, The Jahn-Teller Effect in Molecules and Crystals (Wiley, London, 1972)

[18] H.C. Longuet-Higgins, U. Öpik, M.H.L. Pryce and R.A. Sack, Proc. Roy. Soc. (London) A 2441 (1958)

[19] D.L. Dexter, J. Chem. Phys. 21836 (1953); Phys. Rev. 1261962 (1962)

[20] J.E. Hirsch, Phys. Rev. Letters 541317 (1985)

[21] I.I. Rabi, Phys. Rev. 51652 (1937)

[22] D.J. Moore and G.E. Stedman, J. Phys. A 232049 (1990) 
[23] A. Messiah Quantum Mechanics (North Holland, Amsterdam, 1966) Vol. II, Secs. 41 and 66

[24] D. DiVincenzo and B. Terhal, Physics World March 199853 (1998)

[25] J.I. Cirac and P. Zoller, Phys. Rev. Letters 744091 (1995)

[26] C. Monroe, D.M. Meekhof, B.E. King, W.M. Itano and D.J. Wineland, Phys. Rev. Letters 754714 (1995)

[27] T. Sleator and H. Weinfurter, Phys. Rev. Letters 744087 (1995)

[28] Q.A. Turchette, C.J. Hood, W. Lange, H. Mabuchi and H.J. Kimble, Phys. Rev. Letters 751995 (1995)

[29] S. Somaroo, C.H. Teng, T.F. Havel, R. Laflamme and D.G. Cory, Phys. Rev. Letters 825381 (1999)

[30] B.E. King, C.S. Wood, C.J. Myatt, Q.A. Turchette, D. Leibfried, W.M Itano, C. Monroe and D.J. Wineland, Phys. Rev. Letters 811525 (1998)

[31] H. Schmitzer, S. Klein and W. Dulktz, Physica B 175148 (1991); Phys. Rev. Letters 711530 (1993)

[32] P.W. Shor, Phys. Rev. A 52 R2493 (1995) 